\title{
NECTAR: New Electronics for the Cherenkov Telescope Array
}

C. L. Naumann ${ }^{* a}$, J. Bolmont ${ }^{a}$, P. Corona ${ }^{a}$, E. Delagnes ${ }^{b}$, D. Dzahini ${ }^{c}$, F. Feinstein $^{d}$, D. Gascon ${ }^{e}$, J.-F. Glicenstein ${ }^{b}$, P. Nayman ${ }^{a}$, F. Rarbi ${ }^{c}$, M. Ribó ${ }^{e}$, A. Sanuy ${ }^{e}$, J. Sieiro $^{e}$, J.-P. Tavernet ${ }^{a}$, F. Toussenel ${ }^{a}$, P. Vincent ${ }^{a}$ and S. Vorobiov ${ }^{d, f}$

${ }^{a}$ LPNHE, IN2P3/CNRS Université Paris VI \& IN2P3/CNRS, Paris, France

${ }^{b} I R F U, D S M, C E A$ Saclay, Gif-sur-Yvette, France

${ }^{c} L P S C$, Université Joseph Fourier, INPG \& IN2P3/CNRS, Grenoble, France

${ }^{d}$ LUPM, Université Montpellier II \& IN2P3/CNRS, Montpellier, France

${ }^{e} I C C$-UB, Universitat Barcelona, Barcelona, Spain

${ }^{f}$ DESY-Zeuthen, Zeuthen, Germany

E-mail: haumannelpnhe.in2p3.fr, bolmontein2p3.fr,

coronadlpnhe.in2p3.fr, eric.delagnesdcea.fr, dzahinidlpsc.in2p3.fr,

fabrice.feinsteindeta.in2p3.fr gascondecm.ub.es,

Gean-francois.qlicensteindcea.fr, naymandlpnhe.in2p3.fr,

rarbidlpsc.in2p3.fr, mribodam.ub.es, asanuydecm.ub.es,

sieirode1.ub.es, tavernetdpnhe.in2p3.fr, toussneldenhe.in2p3.fr,

vincentpdin2p3.fn, vorobiovdlpta.in2p3.fr

CTA is the next-generation Cherenkov Telescope Array[四] currently under development by the international CTA consortium[四, based on experience from the three major telescope arrays of the current generation, H.E.S.S., MAGIC and VERITAS. A new kind of front-end hardware, both powerful and flexible, yet inexpensive, will be required to achieve a sensitivity and energy range which is hitherto unprecedented for $\mathrm{TeV}$ gamma rays. One possible solution to equip the up to 100 planned telescopes (with a total of around 100000 photodetector channels) while providing a high degree of flexibility for both triggering and telescope readout is offered by the NECTAr system (New Electronics for the Cherenkov Telescope Array), integrating as much of the front-end functionality as possible into a single ASIC (including pre-amplifiers, fast analog samplers, memory and ADCs). The current status of its development, together with results from measurements and simulations are presented.

International Workshop on New Photon-detectors

June 13-15, 2012

LAL Orsay, France

* Speaker. 


\section{Introduction}

\subsection{The NECTAr project}

$\mathrm{TeV}$ gamma-rays are indirectly detected in a gamma-ray telescope by the Cherenkov light which is emitted in the upper atmosphere (around $10 \mathrm{~km}$ ) from secondary electromagnetic showers. This light flash is detected by an array of optical mirror telescopes, each equipped with around one thousand pixels. The faithful reconstruction of the primary particle's properties necessitates very fast and low-noise readout electronics to properly resolve these faint light pulses, ranging between a few up to several 1000 photons per pixel within a few nanoseconds. Primary requirements are GS/s sampling, a high dynamic range and a short readout time (to counter the optical noise of around $100 \mathrm{MHz}$ per pixel). The development of such camera modules for the future Cherenkov telescope array CTA is the aim of the NECTAr collaboration, which is being financed by the French national research agency ANR and consists of several French and Spanish groups. As parts of the H.E.S.S. or MAGIC collabrations, theses groups can draw upon both hardware designs and experience from these two telescope systems.

\subsection{Modular cameras for CTA}

To achieve a high degree of flexibility as well as simplified production and maintenance, NECTAr-based CTA cameras are separated into semi-autonomous "clusters" of 7 pixels with a shared readout and triggering infrastructure, each containing 3.5" PMTs, a high-voltage board, a front-end board and a backplane. Inter-cluster communication is possible but limited to a transfer of the L0 and L1 trigger signal (see below). Triggering of the telescope is based on a combination of adjacent clusters, and allows for flexible readout options, where local triggering information is used to define when which parts of the camera will be read out. A typical medium-sized CTA camera will contain around 280 such clusters, totalling nearly 2000 pixels.
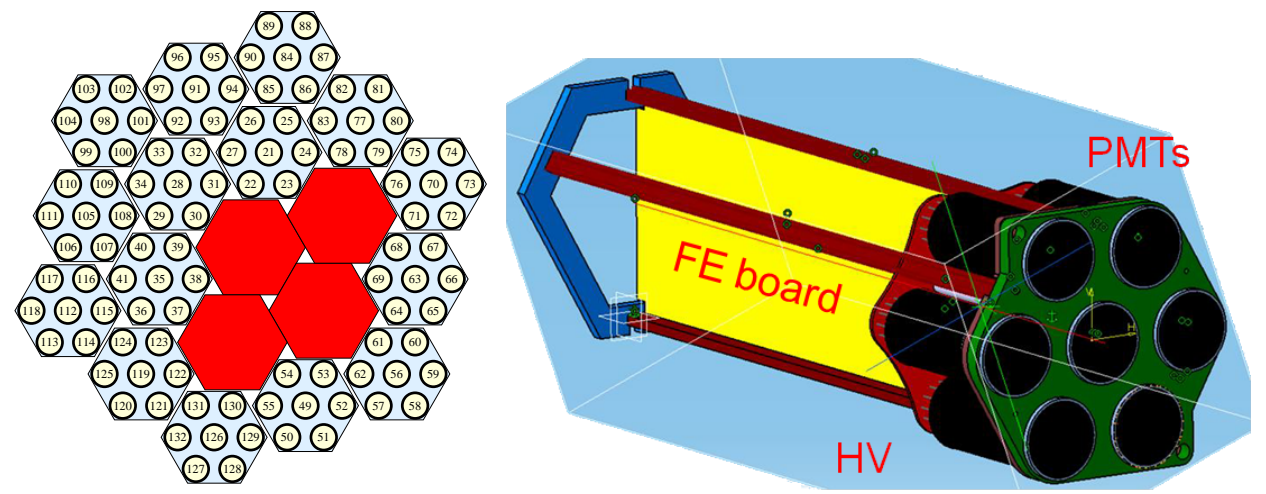

Figure 1: Left: Section of a camera made up of 7-pixel clusters. Highlighted is a possible combination of adjacent clusters used for triggering. Right: sketch of a 7-pixel cluster showing its main components.

\section{The NECTAr board and chip}

At the heart of the NECTAr module lies a single NECTAr readout board, which contains all the amplifiers, analogue memory, ADCs, trigger and readout electronics, as well as communica- 
tions hardware for the cluster (Fig. [1, top). The readout of the cluster is autonomously controlled by an on-board ALTERA FPGA, which also allows signal treatment and data pre-processing. The trigger system is two-tiered: An L0 trigger is formed from the signals of the cluster's own photomultipliers, while the L1 trigger depends on combinations of coinciding L0 triggers from adjacent clusters. Several trigger options are currently under study for NECTAr, including analogue sum or majority triggers (investigated by IFAE, UCM and CIEMAT) or a digital trigger system (developed by DESY).

To further increase the degree of integration, a single ASIC, the NECTAr chip, will incorporate the functions of the pre-amplifiers, the analogue memory and the ADC functions (see Fig. [■, left). Accomodating two to four analogue channels per chip, up to eight chips will be installed on each NECTAr board. The effective dynamic range of each pixel can be increased by splitting the output of a single PMT into two channels, one of which with an additional pre-amplification by a factor of 4-16. Thus, the range from single p.e. resolution on the high-gain channel up to 5000 p.e. on the low-gain channel can be spanned by a single low-cost 12-bit ADC. Prototypes of this ASIC have been developed in the CMOS AMS $0.35 \mu \mathrm{m}$ format. To reduce its power consumption while no data is being read, this NECTAr0 chip uses a power-pulsing scheme.
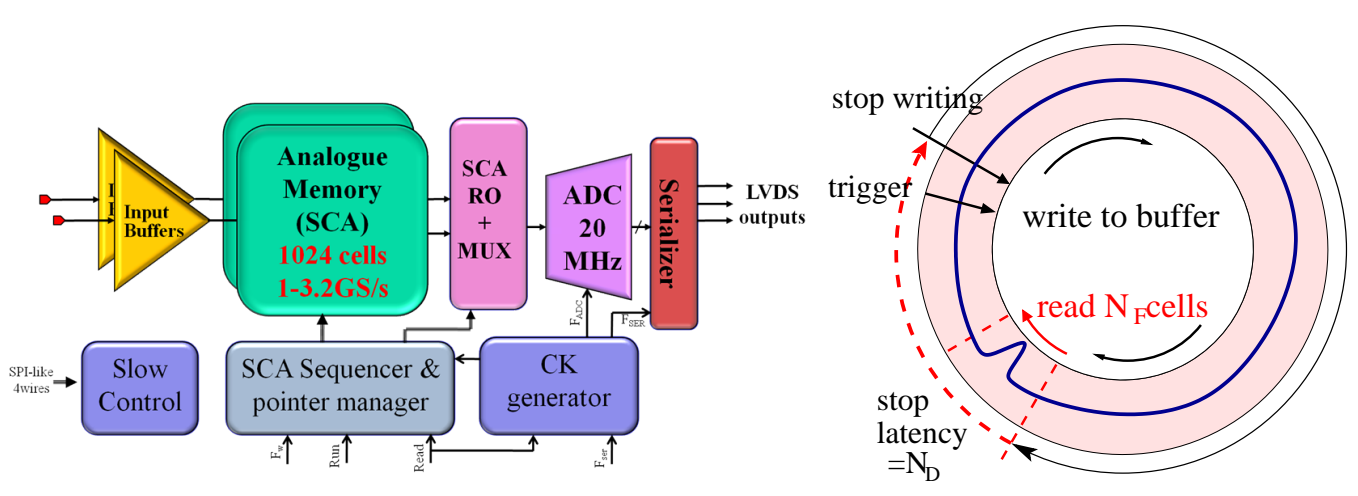

Figure 2: Left: Diagramme showing the functional elements of the NECTAr chip. Right: Cartoon sketch of the analogue ring buffer.

An evolution of the SAM chip employed in the H.E.S.S.-II camera, the analogue ring memory of NECTAr is a switched capacitor array of 1024 bins sampling at a rate of 0.4 to $3.2 \mathrm{GS} / \mathrm{s}$ (corresponding to a total latency from $500 \mathrm{~ns}$ at $2 \mathrm{GS} / \mathrm{s}$ to $2 \mu \mathrm{s}$ at $0.5 \mathrm{GS} / \mathrm{s}$ ). Whenever readout is triggered, writing is stopped and $N_{F}$ bins of the ring buffer are sampled by a $20 \mathrm{MHz}$ ADC, starting at a programmable readout offset $N_{D}$ (Fig. 凹, right). This takes between 2-3.2 $\mu \mathrm{s}$ (for $N_{F}=16$ and 32 , respectively).

\section{NECTAr prototypes for CTA}

The NECTAr group has constructed prototypes of the electronics and structure for a full NECTAr cluster, using a NECTAr board with eight NECTAr0 chips (Fig. B], bottom). The L0 and L1 trigger boards are included as easily-replaceable mezzanines, allowing the test of different triggering schemes; in the final design, they will be included on the board. The system's performance was evaluated with input from either a CTA candidate photomultiplier or a signal generator. 


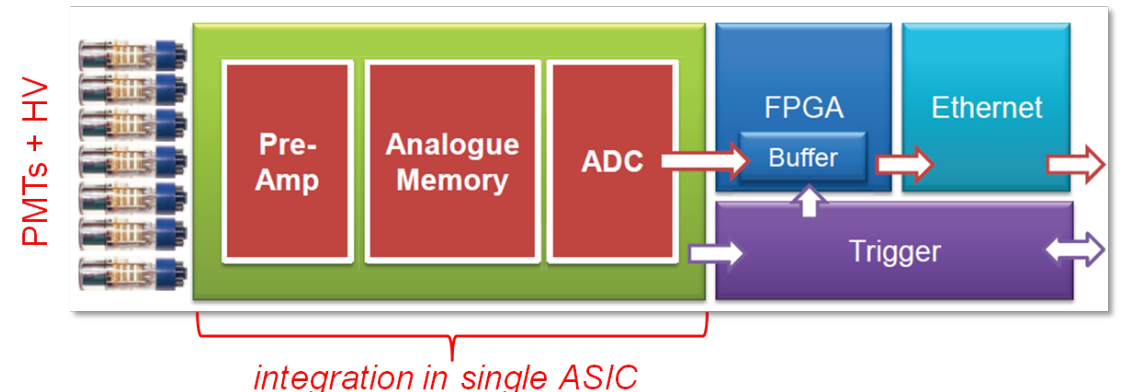

integration in single ASIC

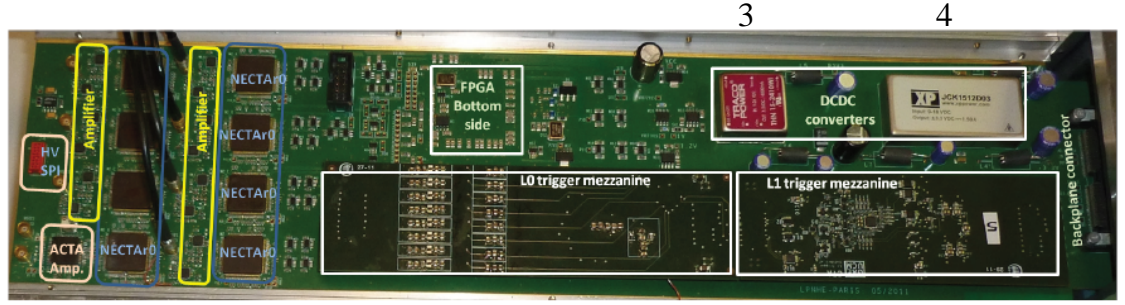

122

5

6

Figure 3: A NECTAr prototype board including ACTA amplifiers (1), NECTAr0 chips (2), ALTERA FPGA (3), DC/DC converters (4), L0 (5) and L1 (6) trigger mezzanine boards.

\subsection{Performance of the prototypes}

Figure 1 , left, shows the photoelectron spectrum measured from a PMT with its gain set at $5 \times 10^{4}$ (to reduce ageing), using a PACTA pre-amplifier[[]]. Due to clear separation between the pedestal and the 1 p.e. peak, a precise calibration of the system gain is possible at run-time.
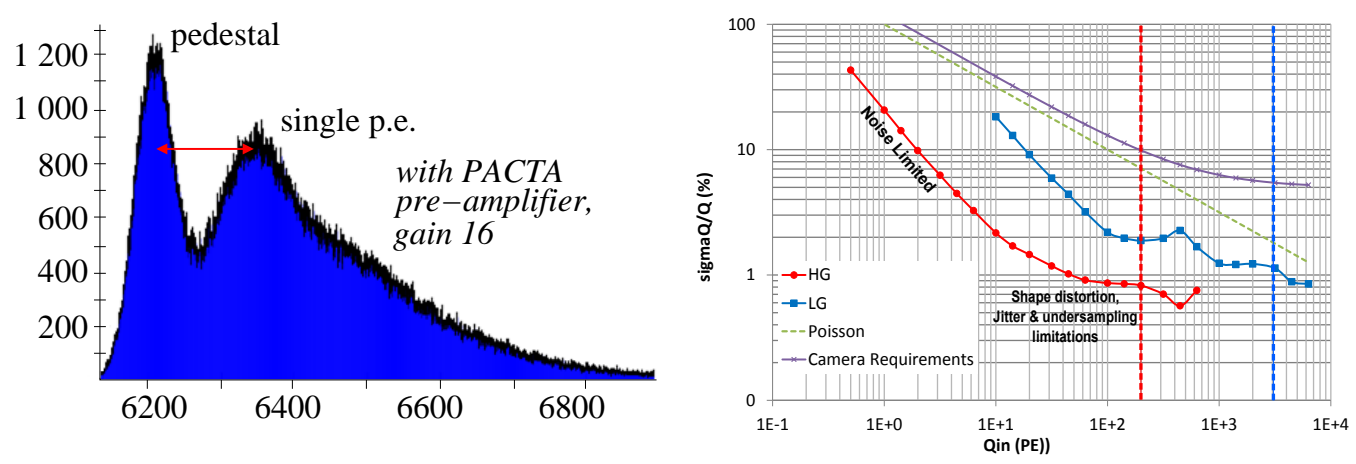

Figure 4: Prototype results. Left: amplitude distribution measured with a weakly-illuminated PMT, using a PACTA pre-amplifier. Right: charge resolution as function of the input amplitude, for the high-gain and low-gain channels, compared to CTA specifications.

The charge resolution (Fig. 团, right) varies from about $20 \%$ on the single p.e. level (where it is noise limited) down to $1-2 \%$ at high charge. This is well below the expected uncertainty from the Poisson distribution of photons at the photocathode, as well as the CTA specifications. With the high-gain channel, high precision measurements are possible up to 200 p.e., while the low-gain channel saturates around 3000 p.e., resulting in a combined dynamic range of 11.3 bit. The $3 \mathrm{~dB}$ 
analogue bandwidth, measured as $410 \mathrm{MHz}$, is well above CTA requirements (300 MHz), while the charge non-linearity is below $2 \%$.

The prototype results are summarised in table $\mathbb{U}$, together with the CTA specifications.

\begin{tabular}{lrrl}
\hline & measured & specification & Unit \\
\hline Nb of channels & 2 & - & diff. channels \\
input impedance & 4 & - & $\mathrm{pF}$ \\
analogue bandwidth & 410 & $\geq 300$ & $\mathrm{MHz}$ \\
memory depth & 1024 & - & $\mathrm{cells}$ \\
sampling frequency & 0.4 to 3.2 & 0.5 to 2 & $\mathrm{GS} / \mathrm{s}$ \\
deadtime (16 bins) & 2 & $\leq 5$ & $\mathrm{~ns}$ \\
ADC LSB & 0.5 & 0.5 & $\mathrm{mV}$ \\
maximum signal & 2 & 2 & $\mathrm{~V}$ \\
dynamic range & 11.3 & $\geq 11$ & $\mathrm{bit} \mathrm{RMS}$ \\
relative non-linearity & $\leq 3$ & $\leq 3$ & $\%$ \\
total noise & $\leq 0.8$ & $\leq 0.8$ & $\mathrm{mV}$ RMS \\
cross-talk & 0.4 & - & $\%$ \\
sampling jitter & $\leq 40$ & $\leq 50$ & $\mathrm{ps} \mathrm{RMS}$ \\
power consumption & 210 & $\leq 300$ & $\mathrm{~mW}$ \\
\hline
\end{tabular}

Table 1: NECTAr protoype performance compared to CTA specifications

\section{Flexible readout: Colibri}

One option that is being tested within the NECTAr framework is the possibility of flexible readout, based on local trigger information. This idea utilises the fact that shower images cover only a small, compact part of the camera, typically less than $10 \%$ for a medium-sized CTA telescope (Fig. [1, left). Thus, by defining readout regions around local L1 triggers (including adjacent
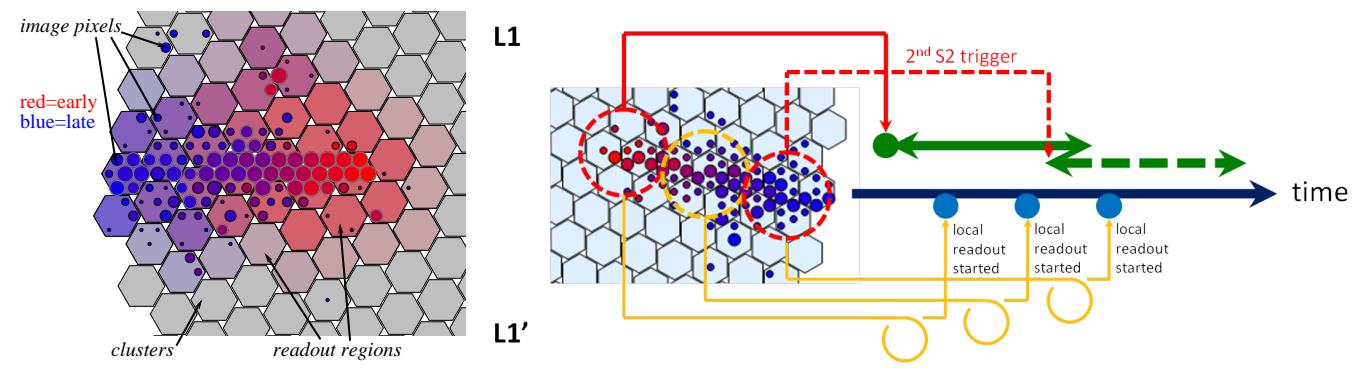

Figure 5: Example for a slowly-developing image of a $15 \mathrm{TeV}$ shower. Colour coding is from red (early) to blue (late), with a total image duration of $55 \mathrm{~ns}$. The colours of the clusters (hexagons) indicate the local triggering and readout times in the case of sliding readout

clusters), the camera's data rate and effective dead-time can be reduced by nearly an order of magnitude. In addition, it is possible to read different parts of the image at different times, allowing the readout to follow the slow development of high-energy showers, avoiding quality losses due to truncated images. Thanks to the high degree of autonomy of the NECTAr clusters, and the local trigger and readout management, such a system can easily be accomodated within the NECTAr 
framework. Figure [1, right, shows a sketch for an implementation of the flexible readout, using a two-threshold trigger with a global threshold L1 and a (lower) local threshold L1'. Triggering at L1 opens a readout enable window (green arrow), during which $\mathrm{L1}^{\prime}$ triggers will start local readout. To compensate for the L1 latency, the L1' signals are locally delayed (loops). Additional L1 triggers can prolong the enable window, $\mathrm{L}^{\prime}$ triggers lying outside the enable window are dropped and create no dead-time, thus L1' thresholds can be set well below L1 for good image coverage.

This system has been implemented for NECTAr - also compatible with the DRAGON hardware - using the analogue L1 trigger cards and the L1 distribution network for generation and treatment of the two signals, as well as for localised readout control. A more detailed description of the concept and its implementation, including simulation results, can be found in [B]].

\section{Summary and outlook}

The NECTAr collaboration is developing high-performance front-end electronics for the nextgeneration Cherenkov Telescope Array CTA, aiming for a high degree of hardware integration, including analogue memory, ADC and amplifiers for one or several pixels in a single ASIC. Prototypes of the chips and boards have been developed and evaluated in a full cluster setup. All specifications by CTA have been reached or surpassed, including a dynamic range up to 5000 p.e. per pixel and readout dead-times below $5 \mu \mathrm{s}$, allowing for trigger rates up to several $\mathrm{kHz}$ at $\mathrm{GHz}$ sampling and a digitisation bandwidth above $400 \mathrm{MHz}$,

The next step will be the design for a NECTAr-based CTA camera, the so-called NECTArCam, the construction of a final demonstrator mini-camera (19-37 modules) in 2013, as preparation for a first full camera. Should the NECTAr design be chosen for CTA, the integration of full NECTAr cameras in the array is foreseen to start in 2015.

\section{Acknowledgments}

We would like to thank the ANR (Agence Nationale de la Recherche) for its support for the NECTAr project.

\section{References}

[1] The CTA consortium, http://www. cta-observatory -org/

[2] The PACTA pre-amplifier, http://iopscience.iop.org/1748-0221/7/01/C01100

[3] C. L. Naumann, L. A. Tejedor and G. Martínez, "Colibri: partial camera readout and sliding trigger for the Cherenkov Telescope Array CTA”, JINST (submitted)

[4] Delagnes E. et al., NIM-A 567 (2006), 21-26

[5] Barcelo M. et al., NIM-A 527 (2007), 382-384

[6] Rarbi F. et al.,IEEE ICICDT Proceedings, Grenoble (2008), 135-138. 